\title{
LOS PRESIDENTES AUTONÓMICOS COMO REGIONAL-MAKERS. EL CASO DE JOSÉ BONO EN CASTILLA-LA MANCHA
}

\author{
THE PRESIDENTES AUTONÓMICOS AS REGIONAL-MAKERS. THE CASE \\ OF JOSÉ BONO IN CASTILLA-LA MANCHA
}

\author{
Manuela Ortega Ruiz \\ Francisco Javier Luque Castillo \\ Universidad de Granada. España/Spain \\ manuelaortega@ugr.es \\ javierluque@ugr.es
}

Recibido/Received: 22/04/2010

Aceptado/Accepted: 07/09/2010

\section{RESUMEN}

En este artículo se quiere realizar una primera aproximación al impacto específico del liderazgo de José Bono en el crecimiento de los sentimientos regionalistas en Castilla-La Mancha, desde la presunción que tales cambios en la cultura política no sólo son resultado de una política sistemática a cargo de las élites castellano-manchegas, sino también una variable explicativa de la hegemonía socialista en dicha Comunidad Autónoma. Para probar nuestras hipótesis, nos centraremos en el discurso del líder, evaluando sus efectos a lo largo del tiempo a través de un análisis concomitante de la evidencia proporcionada por las encuestas de opinión y los resultados electorales.

\section{PALABRAS CLAVE}

Líder, discurso, región, regionalismo.

\section{SUMARIO}

1. Introducción. 2. Comportamiento electoral e identificación regional: datos para una reflexión. 3. El líder como regional-maker. 4. Regional-making en los discursos de José Bono. 5. A modo de conclusión. Bibliografía.

\begin{abstract}
In this article we want to make a first approach to the specific impact of a political leader, José Bono, in the spread of regionalist feelings in Castilla-La Mancha, assuming that such changes in the political culture are not only the result of a systemic policy carried out by the regional elites, but also a explanatory variable of the lasting socialist hegemony. To prove our hypothesis we will focus on the leader's discourse, measuring its effects over time through the concomitant analysis of evidence provided by opinion surveys and election results.
\end{abstract}

\section{KEYWORDS}

Leader, discourse, region, regionalism.

\section{CONTENTS}

1. Introduction. 2. Elections and regional identity: some data to reflect on. 3. The leader as «RegionalMaker». 4. Regional-making in José Bono discourses. 5. By way of conclusion. References. 


\section{INTRODUCCIÓN}

En España, la transición a la democracia conllevó una redefinición del mapa político, es decir, una reorganización territorial del poder. En virtud de dicho proceso se crearon entidades sub-estatales de ámbito regional que, a tenor de lo establecido en la nueva Constitución democrática, estaban llamadas a desarrollar una actividad política autónoma y proactiva en las distintas arenas de poder. Para la formación de estas entidades -las "Comunidades Autónomas" (CCAA)- el marco constitucional dispuso dos mecanismos diferentes: la "vía rápida", pensada para las «nacionalidades históricas»-Cataluña, País Vasco y Galicia-; y la "vía lenta", a la que debieron ajustarse aquellos otros territorios que nunca habían experimentado el autogobierno. El acceso a la autonomía de estos últimos fue antecedido, en cada caso, por un proceso previo de definición territorial que, en no pocas ocasiones, estuvo marcado por la concurrencia de tensiones y controversias. Así ocurrió, paradigmáticamente, con Castilla-La Mancha, calificada en cierto momento por el diputado Ramón Tamames como «el invento más arbitrario de la historia territorial de España» (Asín, 1999: 87). Y es que, como puede inferirse de dicha afirmación, la delimitación territorial de ésta y otras CCAA no pudo inspirarse en referentes del pasado -más o menos remotos-, ni responder a la existencia de un sentimiento identitario regional, espacialmente acotado y diferenciado. Sin embargo -como se tratará de argumentar a lo largo de las siguientes páginas-, ha sido el estímulo y la consolidación de sentimientos regionalistas hasta entonces inéditos -0 , al menos, minoritarios- lo que luego ha hecho posible que «nuevas regiones», como Castilla-La Mancha, alcancen unas cotas de autonomía y protagonismo político inimaginables para los fundadores del Estado de las Autonomías.

Desde nuestro punto de vista, el fenómeno arriba descrito no es casual. Por el contrario, el crecimiento y la consolidación de tales sentimientos regionalistas deben entenderse como resultado de una serie de actuaciones sistemáticas -en los planos material y simbólico- destinadas a forjar y asentar actitudes de identificación emotiva con la región creada. El objetivo inicial de dichas políticas habría sido la legitimación popular de la nueva autoridad regional, pues su establecimiento no constituyó la satisfacción de una demanda social, sino la culminación de un proceso de negociación entre élites. Sin embargo, el impulso activo de la identidad regional -como parte de la tarea más amplia de construcción regionalrepercutió indirectamente en el reforzamiento político de los incumbentes, esto es, de aquellos que ocuparon, por primera vez, los puestos de gobierno regional y -también por primera vezdesplegaron un discurso regionalista.

Asumiendo la importancia que tienen el lenguaje y los discursos en la articulación de procesos sociales, el presente artículo consiste, fundamentalmente, en una exposición sistemática de las evidencias que nos permiten atribuir a los líderes políticos -y más concretamente, a los presidentes de las CCAA- un papel determinante en la configuración de las regiones como marcos de referencia identitaria para sus habitantes. Para acometer esta tarea, la investigación se ha centrado en José Bono, el primer presidente de Castilla-La Mancha. La selección de este caso se explica por varias razones. Por un lado Bono ha permanecido al frente del ejecutivo regional castellano-manchego durante más de dos décadas, por lo que cabe presumirle una notable capacidad de impacto en los procesos políticos de su Comunidad Autónoma. Asimismo, en esta región se ha asistido, desde comienzos de los ochenta hasta nuestros días, a un incremento de las identificaciones regionalistas. Considerando estos hechos, con un primer análisis tentativo de las intervenciones realizadas 
en sede parlamentaria por el Presidente, aquí se pretende proveer de fundamento empírico a la tesis de que el desarrollo del regionalismo en Castilla-La Mancha es consecuencia de una política sistemática orientada a tal fin por parte de sus élites políticas, lo cual habría redundado, indirectamente, en la hegemonía electoral del PSOE en dicha Comunidad.

Aunque la concurrencia de estos fenómenos nos induzca a conjeturar una relación causal entre ellos, la comprobación de tal hipótesis requeriría una mayor exhaustividad en la recogida de información, así como un sofisticado tratamiento estadístico. En este sentido -y como avanzamos anteriormente- el presente trabajo sólo trata de poner de relieve una determinada estrategia política, que se considera relevante para explicar ciertas pautas de conservación y ejercicio del poder en la España contemporánea. Así, dada la modestia de los objetivos que han animado la ejecución de esta investigación, quedan pendientes para más adelante aquellas otras tareas que permitirían arribar a conclusiones relevantes a partir de los presupuestos asentados.

\section{COMPORTAMIENTO ELECTORAL E IDENTIFICACIÓN REGIONAL EN CASTILLA-LA MANCHA: DATOS PARA UNA REFLEXIÓN}

La hipótesis de que el desarrollo del regionalismo en Castilla-La Mancha se debe a una estrategia implementada por las élites regionales -que habría redundado indirectamente en su beneficio político y electoral- encuentra respaldo empírico en los resultados de las elecciones, así como en el material proporcionado por ciertas encuestas de opinión, en concreto, los estudios del Centro de Investigaciones Sociológicas siguientes: Es1475, Es1544, Es1775, Es1820, Es1872, Es2035, Es2146, Es2286, Es2333, Es2455, Es2492, Es2695, Es2286, Es1549, Es1800, Es1905, Es2031, Es2143, Es2286, Es2455, Es2489. En este sentido, y a modo de justificación, se mostrará a continuación una selección de las evidencias que motivaron la realización de este trabajo.

En el caso de Castilla-La Mancha, una región nueva en todos los órdenes (político, administrativo, simbólico, etc.), sus elecciones autonómicas pueden considerarse «una expresión de la evolución de la conciencia de la identidad de los castellano-manchegos como tales, y de su nivel de confianza en la efectividad y la utilidad de las instituciones autonómicas» (Mena, 1998: 179). Desde este punto de vista, la relativamente alta participación registrada en todas las convocatorias -en torno al 75 por ciento-, en conjunción con las sucesivas victorias conseguidas por el PSOE en las mismas, inducen a pensar no sólo en la notable legitimidad alcanzada por el modelo autonómico entre los castellano-manchegos, sino también en el hecho de que tal legitimación ha repercutido en la hegemonía política de los socialistas en las instituciones regionales. Sin embargo, que el PSOE haya sido el único partido capaz de formar gobierno en Castilla-La Mancha, a lo largo de sus casi tres décadas de historia, es un dato que no dice nada por sí solo. Lo relevante, a este respecto, es la circunstancia de que, desde 1996, el Partido Popular (PP) obtiene más votos en la región que el PSOE cuando se trata de elecciones generales.

Dicha distancia entre populares y socialistas llegó a ser de casi doce puntos porcentuales, a favor de los primeros, en las elecciones de 2000, cuando el PP consiguió la mayoría absoluta de los asientos del Congreso de los Diputados. En ese momento los conservadores obtuvieron en Castilla-La Mancha el 52.36 por ciento de los votos, y doce diputados, frente al 40.78 por ciento y los ocho escaños del PSOE. Tal ventaja del PP sobre 
el PSOE en las elecciones a la cámara baja se ha mantenido hasta hoy -si bien en los comicios de 2008 la diferencia fue de 4.85 puntos porcentuales, doce diputados para el PP y ocho para el PSOE-, sin embargo, los conservadores no han conseguido reeditar una mayoría semejante en las elecciones autonómicas.

Gráfico 1. Porcentaje de voto obtenido por el PSOE en Castilla-La Mancha en las elecciones autonómicas y al Congreso de los Diputados (nacionales)

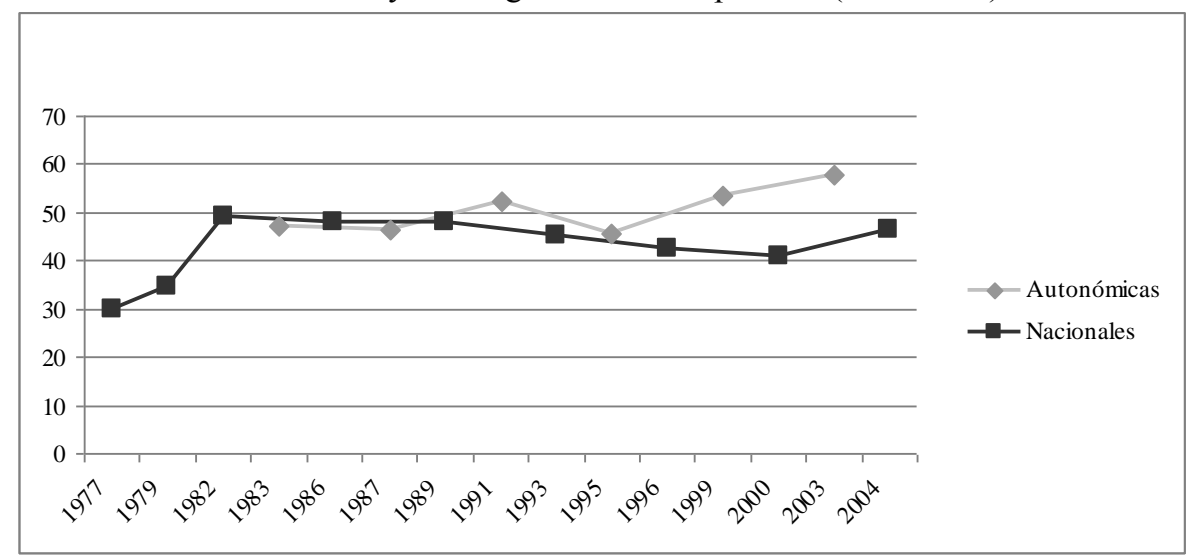

Fuente: Ministerio de Interior

Por el contrario, existe una bolsa de más de cien mil votantes que fluctúa según el tipo de convocatoria electoral, dando lugar a la convivencia en Castilla-La Mancha de dos fenómenos políticos aparentemente contradictorios: una representación regional mayoritariamente conservadora en el Parlamento nacional, y unas instituciones regionales dominadas por los socialistas. Esta circunstancia - que ha llevado a algunos a identificar el comportamiento electoral de los castellano-manchegos con un rasgo de su idiosincrasiaconduce inevitablemente a la reflexión de que el elemento distintivo entre uno y otro proceso lo constituye el hecho de que, en las elecciones autonómicas, el PSOE contó hasta 2003 con el valor añadido de su líder regional y candidato a la Presidencia: José Bono.

Tal hipótesis, congruente con las tesis que apuntan a una personalización de la política en sistemas parlamentarios (McAllister, 2007), no sólo contribuiría a explicar que la misma formación -el PSOE- obtenga en Castilla-La Mancha resultados regularmente dispares, en función del tipo de convocatoria electoral (nacional o autonómica), sino que además encontraría cierto respaldo empírico en la evidencia contenida en algunas encuestas de opinión. Así, por ejemplo, resulta particularmente revelador que José Bono, tras casi veinte años al frente de la Presidencia de Comunidad Autónoma, obtuviera en 2002 la confianza de prácticamente el setenta por ciento de los encuestados (concretamente, el 67.9 por ciento), casi veinte puntos porcentuales más que trece años atrás (cuando se preguntó por primera vez acerca de esta cuestión).

Semejante grado de popularidad, inexplicable en términos de identificación partidaria -pues, como se ha mencionado anteriormente, el PP supera en votos al PSOE en Castilla-La Mancha cuando se trata de elecciones generales o locales- seguramente se relaciona, en gran medida, con el nivel de eficacia percibida. 
Gráfico 2. Confianza en José Bono

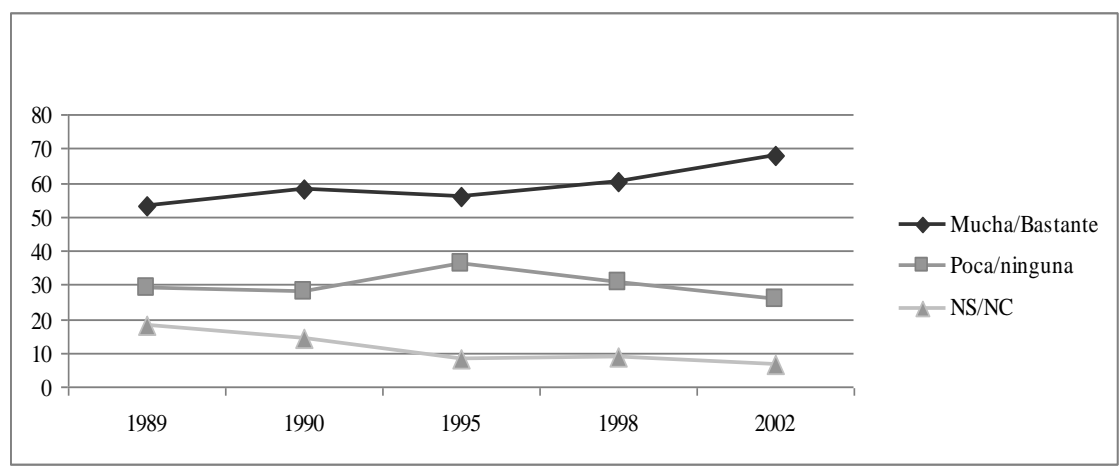

Fuente: estudios del CIS.

En este sentido, como muestra el Gráfico 3, el porcentaje de los que califican la gestión de José Bono como buena o muy buena, no sólo ha crecido siguiendo un patrón parecido al de las categorías alusivas a elevados grados de confianza (Gráfico 2), sino que también ha alcanzado su cénit -con un valor muy aproximado, 68.4 por ciento- el año (2003) en que se pregunta por última vez sobre esta cuestión.

Gráfico 3. Calificación de la gestión de José Bono como presidente de Castilla-La

Mancha

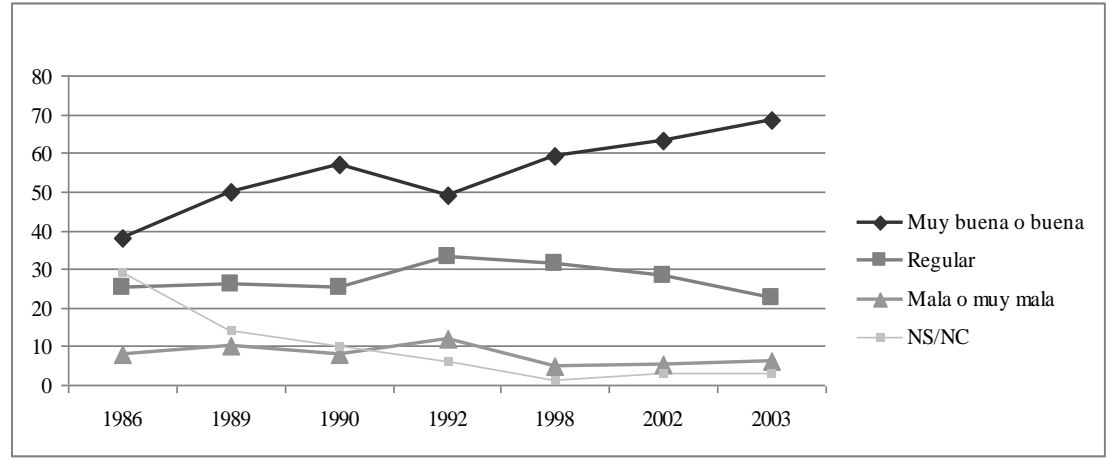

Fuente: estudios del CIS

Sin embargo, la popularidad de Bono - expresada en la confianza o en la calificación a su gestión como presidente- tampoco parece explicar muchas cosas por sí sola. Atribuir a sus cualidades personales, o a las políticas impulsadas desde el Ejecutivo regional, su capacidad de liderazgo -y, por ende, la hegemonía socialista en las instituciones regionalesresulta cuanto menos una aproximación reduccionista, habida cuenta de la orientación conservadora del voto observada en otros niveles de confrontación electoral de Castilla-La Mancha. Así, desde la premisa de que un político atractivo y elocuente, o una acción de gobierno acertada, no son condiciones suficientes para mantener una mayoría electoral durante décadas -sobre todo en una región ideológicamente desplazada a la derecha, a tenor 
de los resultados registrados en los demás comicios-, aquí se aventura la hipótesis de que el liderazgo de Bono en esta región tiene mucho que ver con un tercer fenómeno: el regionalismo.

Gráfico 4. Sentimiento regionalista en Castilla-La Mancha

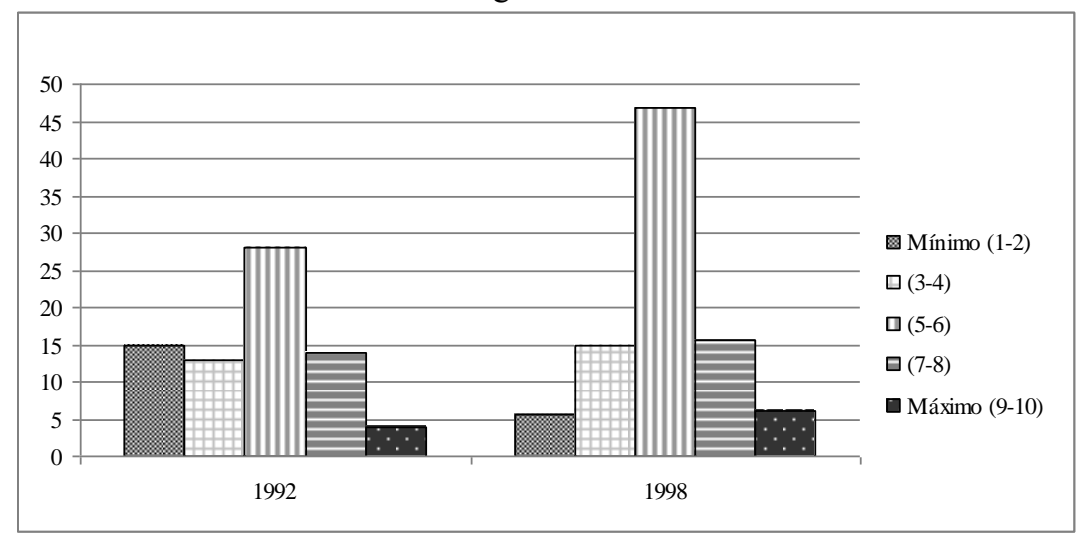

Fuente: estudios del CIS

En Castilla-La Mancha, región nacida -no sin polémica- con la transición a la democracia y el establecimiento del Estado de las Autonomías, los sentimientos regionalistas no sólo eran minoritarios en sus comienzos, sino que además parecieron experimentar un cierto retroceso a finales de la misma década (con la pre-autonomía ya en marcha). Así lo constató García Ferrando (1982:35-36), que detectó un descenso de siete puntos, entre 1976 y 1979 , en el porcentaje de los que se ubicaban en las posiciones altas de la escala de regionalismo subjetivo (del 32 por ciento en 1976 al 25 por ciento en 1979). Casi dos décadas después, y tras quince años de autogobierno -y de ejecutivos liderados por José Bono-, la situación era, sin embargo, bien distinta. Como muestra el Gráfico 4, sólo entre 1992 y 1998, además de descender casi diez puntos el porcentaje de los que manifestaban un sentimiento regionalista mínimo, se incrementó moderadamente el número de los que se situaban en las posiciones más altas de la escala de regionalismo, y creció en casi veinte puntos porcentuales el peso de los que se ubicaban en el nivel intermedio del espectro. Desafortunadamente, no existen datos suficientes para construir una serie temporal que nos permitiera describir con precisión la evolución de los sentimientos regionalistas en Castilla-La Mancha, desde el final de la dictadura hasta nuestros días. No obstante, ciertos indicios nos conducen a pensar que el cambio operado en la cultura política de los castellano-manchegos es estable.

Desde nuestro punto de vista, el desarrollo del regionalismo en Castilla-La Mancha no sólo ha ocurrido en paralelo al crecimiento electoral del PSOE en las elecciones autonómicas, o al aumento de la popularidad de su líder, José Bono; sino que se encuentra además directamente relacionado con estos procesos. En este sentido, y como ya se adelantó en la introducción, el objetivo principal del presente trabajo es realizar una primera exposición, tentativa, de aquellas evidencias que pudieran apoyar la postulación de una relación de causalidad entre la acción del líder -concretamente, en el plano discursivo- y los cambios operados en el comportamiento electoral y la identificación regional de los ciudadanos. 


\section{EL LÍDER COMO REGIONAL MAKER}

La consideración del líder político como regional maker parte de la noción de la región como un proceso abierto y dinámico. Desde esta perspectiva, la existencia de la región no viene dada de un modo predefinido e irreversible, sino que requiere de prácticas muy diversas - a cargo de diferentes instituciones- que contribuyen, de manera continua y cotidiana, a su reproducción como unidad socio-espacial colectiva. Para Paasi (1986), este proceso de institucionalización regional comprende cuatro dimensiones o etapas principales: 1) Asunción de la forma territorial; 2) Desarrollo de la forma conceptual y simbólica; 3) Desarrollo de las instituciones; 4) Reproducción de la región como parte establecida de un sistema y una conciencia regionales. En el caso que nos ocupa, la primera dimensión quedó desarrollada -en lo fundamental- durante los momentos iniciales de la experiencia autonómica, cuando las élites políticas acordaron los límites y la extensión territorial de los nacientes entes regionales. Sin embargo, el avance en la institucionalización regional de Castilla-La Mancha -esto es, la progresión en el conjunto de las etapas- ha consistido en un proceso más prolongado en el tiempo, posible en virtud de la intervención de múltiples actores políticos y sociales.

En términos generales, instituciones como la Universidad o el propio gobierno, u organizaciones como las de representación de intereses empresariales o los sindicatos, pueden ejercer de regional makers, no obstante, varios autores han destacado la importancia de ciertos actores para la consolidación de la región, como ente definido, dentro del imaginario colectivo. Jordan (1996: 107-108), por ejemplo, resaltó el papel desempeñado por los medios de comunicación, las empresas y los políticos en la difusión de las identidades regionales. En el caso de Castilla-La Mancha, al tratarse de un proceso políticamente vertebrado de arriba abajo (top down), la construcción regional fue especialmente promovida por la clase política surgida tras su constitución en CCAA. La supervivencia política de estas nuevas élites regionales dependía, en última instancia, de que la población castellanomanchega aceptaran la incorporación de un nuevo nivel de gobierno en su universo político, y el primer mandatario de Castilla-La Mancha tenía los recursos -además de la motivaciónpara convertir aquel objetivo en realidad.

Habida cuenta de lo mencionado más arriba, la labor de construcción regional llevada a cabo en Castilla-La Mancha habría de evidenciarse, principalmente, mediante el examen de la acción política desarrollada por el gobierno autonómico. Sin embargo, dado que la acción política encuentra expresión tanto en las políticas públicas como en el discurso de los dirigentes, el presente trabajo se ha centrado en la segunda manifestación, desde la convicción de que un fenómeno postmoderno, como la emergencia de entes e identidades regionales, merece una primera aproximación postmoderna, como la que constituye el análisis del discurso (Howarth, 1995).

Finalmente, si se ha privilegiado el discurso del presidente autonómico -en detrimento de otros discursos que seguramente han contribuido, de igual forma, a la construcción regional de Castilla-La Mancha-, ha sido porque ciertas evidencias -que ya fueron presentadas en el segundo apartado- nos inducen a pensar en José Bono como un líder político con una extraordinaria capacidad de impacto sobre los castellano-manchegos. La comprobación de este último presupuesto, por otra parte, no haría sino confirmar la reproducción, en el sub-sistema regional, del esquema de presidencialización del proceso político ya observado en el nivel nacional (Biezen \& Hopkin, 2005). 


\section{REGIONAL-MAKING EN LOS DISCURSOS DE JOSÉ BONO.}

Estrategias retóricas para el regional-making

Según Jacobo García (2003), cualquier discurso que tome como referencia principal un territorio, contribuye a su objetivación colectiva e intersubjetiva, no sólo en un sentido comunicativo, sino también movilizador. Sin embargo, el "territorio" puede emerger en el discurso a través de diferentes estrategias retóricas:

a. La "naturalización": consiste en la presentación como hechos "naturales" -o como la consecuencia inexorable de fenómenos naturales- de fenómenos creados social o históricamente.

b. La "territorialización": constituye la estrategia a través de la cual el territorio emerge como base de clasificación e identificación de la comunidad política imaginada. Con esta estrategia se ocultan o desplazan a un plano secundario los conflictos sociales internos, así como otras bases posibles de identificación (la clase social, la provincia, la comarca, etc.) que pudieran existir dentro de la comunidad.

c. La «eternización»: supone la sustracción, a los fenómenos socio-históricos, de su carácter histórico, de tal manera que son presentados como permanentes e inalterables. Esta estrategia se concreta mediante la alusión a fenómenos de tipo biológico o natural como elementos constitutivos de la identidad territorial, o bien mediante la elaboración de una narrativa histórica propia en la que costumbres y tradiciones adquieren un carácter inmemorial.

d. La búsqueda antitética de un "Otro": esta estrategia pondría de manifiesto que la construcción de la identidad regional pasa, necesariamente, por la conjugación de dos discursos complementarios: el de la "integración" ("nosotros-aquí") y el de la "diferencia" ("ellos-allí).

Dado que los regionalismos - como los nacionalismos-son "ideologías territoriales" (Anderson, 1988: 26-27), consideramos oportuno abordar los discursos de José Bono a partir de las categorías expuestas anteriormente. De esta manera, no sólo se pretendía evidenciar la orientación regionalista informadora de su narrativa, sino que además se esperaba desentrañar las nociones subyacentes sobre su región, presumiblemente más próximas al "nuevo regionalismo" que al "regionalismo tradicionalista". El "nuevo regionalismo", que nace en Europa occidental tras la II Guerra Mundial, se fundamenta sobre bases funcionales y operativas. A diferencia del "regionalismo tradicionalista" propio del siglo XIX (foralista, conservador, etnicista, romántico), este regionalismo pretende dar respuesta a problemas que afectan a las necesidades básicas de la sociedad en su conjunto. Una ordenación del territorio más eficaz, el desarrollo de políticas económicas adaptadas a la realidad regional particular, o el desbloqueo de la crisis del sistema de gobierno burocrático-centralizado, son algunas de las exigencias más reiteradas por los nuevos regionalistas (García, 2003).

\section{El discurso de José Bono}

Las cuarenta y tres intervenciones parlamentarias de José Bono que han sido objeto de análisis para esta investigación, correspondientes al período 1995-2004 evidencian que el ex Presidente de Castilla-La Mancha hizo un uso frecuente de las estrategias retóricas expuestas en el anterior apartado, al objeto de promover y afianzar una determinada visión regionalista de la realidad política. Obviando el hecho de que privilegia a la región, 
sistemáticamente, como base de clasificación de la comunidad política -lo cual confiere a la territorialización el rango de estrategia principal-, Castilla-La Mancha adquiere entidad propia en su discurso, de manera recurrente, como un territorio históricamente maltratado por el centralismo:

"La existencia de la Comunidad Autónoma, si me permiten, ha sido providencial para una Región históricamente condenada a la incuria de los viejos regímenes autoritarios y centralistas. La autonomía nos ofreció una oportunidad y los castellano-manchegos hemos sabido aprovecharla, y hoy Castilla-La Mancha pesa en España más que ha pesado en toda su historia" (DS Cortes de Castilla-La Mancha, 10 octubre 2000).

Para José Bono, la superación de esa dependencia de los antiguos centros de poder, que habría condenado en el pasado a Castilla-La Mancha al subdesarrollo, debe ser celosamente preservada, en la nueva etapa autonómica, de la amenaza que constituyen los intereses particulares. En este sentido, al socialista le gusta recordar con frecuencia episodios de su vida política en los que, a su juicio, ha antepuesto los intereses de CastillaLa Mancha a su lealtad partidaria:

"Una visión estrechamente partidista quizá me hubiese aconsejado alimentar la oposición entre el Gobierno Socialista de Castilla-La Mancha y el Gobierno de derechas del señor Aznar. Pero hemos llegado a acuerdos con el señor Aznar y con su Gobierno, con la misma firmeza que rechazamos algunos otros de Felipe González. Precisamente ayer lo hablaba con él, y aunque no le gusta que diga esto, se lo comenté... Es evidente, que defender a Aznar o a González no es defender a Castilla-La Mancha; son cosas bien distintas" (DS Cortes de Castilla-La Mancha, 18 octubre 2001).

Como puede colegirse de estas últimas palabras, la autonomía de Castilla-La Mancha, desde la óptica de José Bono, implica un talante reivindicativo, lo cual conlleva en ocasiones la explotación del agravio comparativo con otras regiones. Así, Castilla-La Mancha no sólo se define en relación al centro que la subyugaba en tiempos pasados, sino también respecto al resto de regiones españolas y, en especial, respecto a las que Bono considera históricamente privilegiadas. En este sentido, Cataluña, y más concretamente quien fuera su Presidente durante más de veinte años, Jordi Pujol, aparece habitualmente -a colación de unas declaraciones polémicas-en las alocuciones de José Bono, al fin de ilustrar el tipo de comportamientos y actitudes que, en su opinión, pretenden relegar a Castilla-La Mancha a un segundo plano, rebajando en consecuencia la dignidad de su gente:

"A mí me han elegido para defender a Castilla-La Mancha y no es que quiera menospreciar a nadie, pero a quien nos menosprecia no estoy dispuesto a defenderlo. Por ejemplo, cuando el señor Pujol, por ponerle un ejemplo lejano, menosprecia a Cuenca, no tengo que esperar a que desde mi partido en Madrid salga alguien a defender la provincia, tengo que salir yo" (DS Cortes de CastillaLa Mancha, 20 agosto 2002).

De hecho, en cierta ocasión, José Bono opone explícitamente la identidad de Castilla-La Mancha a ciertas ideas sobre dicha región manifestadas, muchos años atrás, por Jordi Pujol y el dirigente histórico catalán Josep Tarradellas: 
"Decía Tarradellas, cuando vino un año a la Feria de Albacete: 'Los autonomistas de esta tierra caben en un taxi y sobran plazas', y añadiría lo que luego dijo Pujol: 'Cataluña no es Castilla-La Mancha'. Ya les he contestado suficientemente. Simplemente evoco estas citas para decirles que hemos hecho lo más difícil, que ha sido conseguir una identidad regional que no teníamos, y ahora queda lo más importante" (DS Cortes de Castilla-La Mancha, 25 junio 2003).

Sin embargo, José Bono no sólo concibe Castilla-La Mancha como una región ignorada por el centro, o menospreciada por los catalanes. En su búsqueda antitética de «otros», a partir de los cuales delimitar a los propios («nosotros»), tampoco vacila a la hora de apuntar con el dedo a aquellas regiones que lesionan los intereses de Castilla-La Mancha, especialmente en lo relativo al agua:

"Desde otra perspectiva, sin ánimo demagógico y reconociendo que el deporte del golf produce empleo y atrae turismo, quiero mostrarles el anuncio de la Generalitat Valenciana en que se afirma: 'Presentamos la última moda en golf. El golf está de moda en la Comunidad Valenciana. Aquí puede ver 16 campos del golf...' Pues bien, en Castilla-La Mancha no tenemos ni un solo campo de golf porque hemos creído conveniente atender otras necesidades más prioritarias antes que regar campos de golf que tanta agua consumen y tan escasa la tenemos" (DS Cortes de Castilla-La Mancha, 8 agosto 1995).

$\mathrm{Y}$ es que las cuestiones medioambientales han provisto a José Bono de un material muy sustancioso para elaborar imaginería regionalista. De este modo, Cabañeros, Hoces de Cabriel, o Tablas de Daimiel -entre otros lugares- se han convertido en espacios emblemáticos del regionalismo castellano-manchego, pero no como resultado de una exaltación sistemática de su belleza natural -como probablemente correspondería a una estrategia retórica de naturalización- sino por constituir escenarios en los que la defensa de lo propio ha vencido frente a la voluntad insolidaria de intereses ajenos al terreno.

"El Trasvase se construyó para regar las tierras de Murcia, no las castellanomanchegas, y para ello se prohibió su uso a lo largo del recorrido del acueducto por nuestra región. Durante estos últimos años, el Gobierno Regional, ha conseguido, al menos, dos cosas importantes: que se pueda utilizar el acueducto para llevar agua a las Tablas de Daimiel -con el voto contrario del Partido Popular, cuando se planteó la primera iniciativa-, y que se pueda abastecer a Ciudad Real y Puertollano utilizando el Trasvase" (DS Cortes de Castilla-La Mancha, 28 enero 1997).

Pero José Bono no se limita en sus discursos a crear región mediante las estrategias retóricas de la territorialización o la alteridad. A lo largo de sus intervenciones, Bono va modelando un arquetipo de castellano-manchego, un ideal de ser regional en el que descuellan los valores de la hospitalidad, la amabilidad, la generosidad, o la lealtad a la palabra dada. Asimismo, el dirigente autonómico enfatiza en un sentido no etnicista- la línea que separa a los castellano-manchegos de los que no lo son, recurriendo para ello a la por él odiada figura del "cunero":

“No consideraron oportuno que dijese que en esta tierra no sobra nadie, haya nacido en Casablanca o en Sebastopol, pero sobran todos los que habiendo nacido aquí no sienten los colores de esta tierra y les de igual salir en la 'uno' que no 
salir, o que esta tierra tenga agua o no la tenga? ¿Les pareció mal que dijera que a partir de ahora pienso ser beligerante con los cuneros, cualquiera que sea su filiación política? ¿Les incomodó que no citase, como cuneros de renombre, a Rupérez y a Tocino? Pues si les incomodó, les va a seguir molestando, porque pienso ser beligerante con quienes creen que esta Región es un distrito electoral cómodo para ser Diputado o Senador, porque está cerca de Madrid. Mi gusto sería que a las próximas elecciones ningún cunero pudiese aparecer por aquí, porque tuviese el estigma vergonzoso de haber venido a buscar distrito, sin ofrecer nada a cambio" (DS Cortes de Castilla-La Mancha, 9 junio 1998).

Las evidencias hasta ahora seleccionadas parecen sugerir que José Bono, mientras estuvo al frente del Gobierno de Castilla-La Mancha, impulsó una estrategia regionalista que encontraba expresión en el plano discursivo. Sin embargo, lejos de constituir parte de una hipotética «agenda oculta», el fomento del regionalismo fue siempre un objetivo político abiertamente reconocido por el líder socialista en diversos momentos. En 1995, explicaba en los siguientes términos el surgimiento de la conciencia regional:

"En el caso de Castilla-La Mancha, como en el de otras regiones españolas en las que el regionalismo carece de base histórica, no ha sido la función -el sentimiento regional- la que ha creado el órgano -la autonomía-, por usar un símil biológico predarwinista. Antes al contrario, ha sido el órgano el que ha creado la función. Ha sido la autonomía la que ha traído la conciencia regional. Y este es un proceso que no admite parada ni retroceso" (DS Cortes de Castilla-La Mancha, 29 junio 1995).

\section{A MODO DE CONCLUSIÓN}

Como se dijo en la introducción, el objetivo de este trabajo no era otro que la exposición sistemática de las evidencias que nos permitieran atribuir a los líderes políticos -y más concretamente, a los Presidentes de las CCAA- un papel determinante en la configuración de las regiones como marcos de referencia identitaria para sus habitantes. Para acometer dicha tarea se escogió el caso de José Bono, máximo dirigente regional, durante más de dos décadas, en Castilla-La Mancha. La elección de este caso se justificó por la concurrencia, durante la mayor parte del período correspondiente a la experiencia autonómica, de tres fenómenos significativos: la re-elección continuada de su primer Presidente autonómico, la hegemonía electoral del PSOE en las elecciones regionales, y el crecimiento de los sentimientos regionalistas.

Adoptando un enfoque postmoderno y postestructuralista, se optó por analizar los discursos de este líder, desde la presunción de que en ellos encontraría respuesta -al menos parcial- nuestro interrogante acerca de la responsabilidad del presidente autonómico en el desarrollo del regionalismo en su Comunidad. En este sentido, el propósito de las presentes páginas se puede considerar satisfecho, pues el examen de las intervenciones parlamentarias realizadas por José Bono, a lo largo de su mandato presidencial, ha permitido acreditar su condición de regional-maker, no sólo por el hecho de que en él sea frecuente el empleo de estrategias retóricas orientadas a la promoción de una concepción regionalista de la realidad política, sino porque él mismo reconoce que el fomento de la conciencia regional constituye una prioridad para su Gobierno. 
Este hallazgo, en realidad, lejos de zanjar asunto alguno, no hace sino plantear nuevas preguntas en torno a la relevancia de la identidad para la conservación y el mantenimiento del poder en algunas regiones de España. En cualquier caso, y aunque quede pendiente la comprobación de la relación de causalidad, este trabajo aporta nuevos y sólidos indicios para apoyar la hipótesis de que, en las Comunidades Autónomas, el liderazgo y la identidad regional constituyen variables explicativas de otros procesos políticos.

\section{BIBLIOGRAFÍA}

ANDERSON, J. (1988): "Nationalist ideology and territory" en Johnston, R.J. et al (eds.), Nationalism, self-determination and political geography, London, Croom Helm, pp. 18-39.

ASÍN VERGARA, R. (1999): El nacimiento de una región: Castilla-La Mancha 1975 - 1995, Madrid, Biblioteca Añil

BIEZEN, I. y HOPKIN, J. (2005): "The presidentialization of Spanish democracy: sources of primerministerial power in post-Franco Spain" en Poguntke, T. y Webb, P.: The presidentialization of politics: a comparative study of modern democracies, Oxford, Oxford University Press, pp. 106-127.

GARCÍA ÁLVAREZ, J. (2003): Provincias, regiones y comunidades autónomas: la formación del mapa político de España, Madrid, Papeles del Senado.

GARCÍA FERRANDO, M. (1982): Regionalismo y autonomía en España, 1976-1979, Madrid, CIS. HOWARTH, D. (1995): "La teoría del discurso" en Marsh, D. y Stoker, G. (eds.): Teoría y métodos de la ciencia política, Madrid, Alianza Editorial, pp. 125-141.

JORDAN, T. (1996): "Recent German research on space-related attachments and regional identities", Geografiska Annaler, vol. 78 B (2), pp. 99-111.

McALLISTER, I. (2007): "The Personalization of Politics" en Dalton, R. J. y Klingemann, H.D.: The Oxford Handbook of Political Behaviour, Oxford, Oxford University Press, pp. 571-588.

MENA MARTÍNEZ, L. (1998): "Castilla-La Mancha. Elecciones autonómicas” en Alcántara, M. y Martínez, A.: Las elecciones autonómicas en España, 1980-1997, Madrid, CIS, pp. 179-202.

PAASI, A. (1986): "The institutionalization of regions: a theoretical Framework for understanding the emergence of regions and the constitution of regional identity" en Fennia, vol. 164 (1), pp. 105-146.

\section{Breve currículo:}

\section{Manuela Ortega Ruiz}

Investigadora predoctoral FPU, Universidad de Granada. Desarrolla su tesis doctoral en el Departamento de Ciencia Política y de la Administración. Entre sus publicaciones recientes destaca el artículo en colaboración con Francisco Javier Luque Castillo "Obama es el mensaje. Una interpretación macluhaniana del nuevo liderazgo presidencial estadounidense", para la Revista de Psicología Política $\mathrm{n}^{\mathrm{o}} 39$ (noviembre de 2009). También ha colaborado con el mismo autor en la obra Estudios de Ciencia Política y de la Administración (Valencia, Tirant lo Blanch, 2009), con un capítulo titulado "Perfil sociodemográfico de los altos cargos de la Junta de Andalucía: una aproximación a la élite político-administrativa andaluza".

\section{Francisco Javier Luque Castillo}

Investigador predoctoral FPU, Universidad de Granada. Doctorando en el Departamento de Ciencia Política y de la Administración. Entre sus publicaciones recientes destaca el artículo conjunto con Manuela Ortega Ruiz "Obama es el mensaje. Una interpretación macluhaniana del nuevo liderazgo presidencial estadounidense", para la Revista de Psicología Política n 39 (noviembre de 2009). También han colaborado en la obra Estudios de Ciencia Política y de la Administración (Valencia, Tirant lo Blanch, 2009), con un capítulo titulado "Perfil sociodemográfico de los altos cargos de la Junta de Andalucía: una aproximación a la élite político-administrativa andaluza”. 